CLINICOPATHOLOGICAL CONFERENCE

\title{
Progressive neuropsychiatric problems following institution of highly active antiretroviral therapy
}

\author{
R F Miller, S S Dave, J W Tang, P J Shaw, H R Jäger, E G L Wilkins
}

Sex Transm Infect 2005;81:351-357. doi: 10.1136/sti.2004.014027

\begin{abstract}
A 31 year old HIV infected woman developed neuropsychiatric problems soon affer starting highly active antiretroviral therapy (HAART). Despite modifying and subsequently stopping HAART her condition progressively worsened. Cranial magnetic resonance imaging revealed multiple areas of abnormal signal suggestive of either a vasculitis or demyelination.
\end{abstract}

See end of article for authors' affiliations

Correspondence to: Dr R F Miller, Mortimer Market Centre, RFUCMS, University College London, London WC1E 6AU, UK; rmiller@gum.ucl.ac.uk

Accepted for publication 29 November 2004

\section{CASE PRESENTATION \\ (Dr R F Miller and Dr S S Dave)}

A 31 year old black African woman was admitted to hospital in late October 2003. She gave a 3 week history of low mood, tearfulness, sleepiness, and easy fatiguability. The patient had arrived in the United Kingdom 4 months previously, at which time she had been found to be HIV-l antibody positive. At the end of September 2003 her CD4 count was 50 cells $\times 10^{6} / 1$ and HIV viral load was 17300 copies $/ \mathrm{ml}$. Three weeks before admission the patient had started antiretroviral therapy with a combination of zidovudine, lamivudine, and efavirenz and had started co-trimoxazole, as primary prophylaxis against Pneumocystis pneumonia. She had no medical history of note.

On admission to hospital additional history was obtained from the patient. Her symptoms had begun immediately after starting antiretroviral therapy. She further described a persistent mild frontal headache, without relieving or exacerbating factors. She denied experiencing nightmares, speech difficulties, problems with activities of daily living, or loss of consciousness. Systematic inquiry was negative.

On examination the patient looked well, she had a flat affect, and was alert and orientated. There were no signs of meningism and in the limb tone, power, reflexes, coordination, and sensation were intact. Funduscopy was normal. She was apyrexial; there was no peripheral lymphadenopathy. Examination of the mouth and skin was normal. In the cardiovascular system the pulse was 70 and regular, and blood pressure 115/70 mm Hg. Examination of the chest, abdomen and joints was normal. Initial investigations showed normal urea and electrolytes, lactate, liver function, and thyroid function tests. The C reactive protein (CRP) was 25 (normal <5) IU/l. A full blood count showed haemoglobin $(\mathrm{Hb}) 10.8 \mathrm{~g} / \mathrm{dl}$, white blood cells (WBC) $3.2 \times 10^{9} / 1$ (neutrophils $\left.=1.7 \times 10^{9} / 1\right)$, and platelets $353 \times 10^{9} / 1$. A sickle screen was negative and haemoglobin electrophoresis was normal. The CD4 count was 100 cells $\times 10^{6} / 1$ and the HIV viral load was 90 copies $/ \mathrm{ml}$. In blood serology for syphilis and toxoplasmosis and a cryptococcal latex agglutination (CRAG) test were negative. Hepatitis serology showed a positive hepatitis B surface and e antigen, consistent with a high infectivity hepatitis B carrier. A chest radiograph was normal.

The patient's symptoms were ascribed to her antiretroviral therapy and nevirapine was substituted for efavirenz. This change of therapy had no impact on her symptoms.

What thoughts do you have on this patient's presentation and initial management? Are any further investigations indicated at this stage?

\section{DISCUSSION \\ (Dr E G L Wilkins)}

As presented, the history is consistent with efavirenz induced central nervous system (CNS) side effects, coming on immediately after commencing highly active antiretroviral therapy (HAART) and persisting for the first 3 weeks. However, the absence of vivid dreams/nightmares (present in $85 \%$ ), the presence of headache and the flat affect (both unusual with efavirenz toxicity and more suggestive of organic brain disease), and the persistence of these symptoms beyond 3 weeks, argue strongly against this. Although plasma efavirenz levels remain detectable for over 2 weeks in some people of African descent after withdrawal, the lack of improvement on switching to nevirapine is another factor suggesting another cause. On the other hand, there is very little else to point towards primary or secondary brain pathology with no focal signs on examination, no fever, no lymphadenopathy and, with the exception of mild anaemia and leucopenia (both consistent with advanced HIV), no pointers to an HIV related opportunistic infection or tumour. The negative CRAG, syphilis, and toxoplasma

\footnotetext{
Abbreviations: AAFB, alcohol and acid fast bacilli; ADEM, acute disseminated encephalomyelitis; CMV', cytomegalovirus; CRP, $C$ reactive protein; CSF, cerebrospinal fluid; CT, computed tomography; DSA, digital subtraction angiography; EBV, Epstein-Barr virus; FSE, fast spin echo; HAART, highly active antiretroviral therapy; HSV, herpes simplex virus; IRIS, immune reconstitution inflammatory syndrome; MAC,

Mycobacterium avium complex; MRI, magnetic resonance imaging; NNRTI, non-nucleoside reverse transcriptase inhibitor; NRTI, nucleoside reverse transcriptase inhibitors; PML, progressive multifocal
}

leucoencephalopathy; VZV, varicella zoster virus 
serology, although not excluding CNS infection with these agents, make their diagnosis extremely unlikely. Of note, the patient's baseline CD4 was 50 cells $\times 10^{6} / 1$ and it doubled after being on HAART for 3 weeks with an equally dramatic fall in HIV viral load (from 17300 copies $/ \mathrm{ml}$ to 90 copies $/ \mathrm{ml}$ ). Given her ethnic background and her advanced HIV, there is a real possibility of meningeal/disseminated tuberculosis despite the absence of any focal or meningeal features. Hence, imaging of the CNS with magnetic resonance imaging (MRI) would be my next step. This would be followed by lumbar puncture with manometry with cerebrospinal fluid (CSF) being sent for standard cell count and biochemistry, India ink, CRAG, treponemal antibody, Gram, Ziehl-Neelsen stain, and standard culture. In addition, CSF should be sent for cytology as well as nucleic acid detection testing for herpes simplex virus (HSV), varicella zoster virus $(\mathrm{VZV})$, cytomegalovirus (CMV), Epstein-Barr virus (EBV), JC virus, enterovirus, Toxoplasma gondii, and Mycobacterium tuberculosis.

\section{CASE PRESENTATION}

\section{(Dr Miller and Dr Dave)}

A computed tomography (CT) scan of the brain was normal. At lumbar puncture the opening pressure was normal; microscopy showed <l lymphocyte, and no organisms. Cytological examination showed no malignant cells. The CSF protein was $0.3 \mathrm{~g} / \mathrm{l}$ and CSF: plasma glucose ratio was 3.4:5.2. In CSF the CRAG, syphilis and toxoplasma serology were negative; PCR for human herpes viruses 1-5 (HSV type 1 and 2, VZV, CMV, and EBV) and JC virus was negative. Culture of CSF for bacteria, mycobacteria, and fungi was negative.

After 9 days in hospital the patient's symptoms remained unchanged. The haemoglobin had fallen to $9.0 \mathrm{~g} / \mathrm{dl}$; this was ascribed to zidovudine, which was stopped and tenofovir was commenced. The patient was discharged the following day, taking tenofovir, lamivudine, and nevirapine. She was readmitted 7 days later and gave a week's history of nausea and vomiting, which had begun after tenofovir had been commenced. In addition, the patient reported a 2 day history of unsteadiness on her feet and a fall. Over the 24 hours before re-admission her headache had become more severe.

Examination revealed she was alert and orientated, but was emotionally labile. General examination was normal; blood pressure 125/80 mm Hg. Neurological examination showed a right sided pyramidal weakness (MRC grade $=4$ / 5), apraxia in the right arm, generalised hyper-reflexia, more marked on the right, bilateral flexor plantar reflexes, and dysdiadokokinesis in the right hand. Sensation was intact. The patient had a scanning dysarthria but no nystagmus. Funduscopy showed bilateral "flame-shaped" vascular haemorrhages, there was a right sided upper motor neuron seventh cranial nerve palsy: the other cranial nerves were normal. There was no meningism.

Investigations showed normal urea and electrolytes, liver function tests and lactate; the CRP was $20 \mathrm{IU} / \mathrm{l}$. A full blood count showed $\mathrm{Hb} 10.4 \times 10^{9} / \mathrm{l}$, WBC $2.2 \times 10^{9} / \mathrm{l}$ (neutrophils $=0.8$ ) , and platelets $227 \times 10^{9} / \mathrm{l}$. Urinanalysis was normal. Culture of blood and urine for bacteria, mycobacteria, and fungi was normal. An ECG was normal. A magnetic resonance (MR) scan of the brain was performed.

\section{IMAGING}

\section{(Dr H R Jäger)}

The MR appearances (fig l) show involvement of supratentorial and infratentorial white matter, and suggest a demyelinating process or a pathology affecting the small perforating vessels supplying the white matter. The latter includes a vasculitic process. In the absence of hypointensity
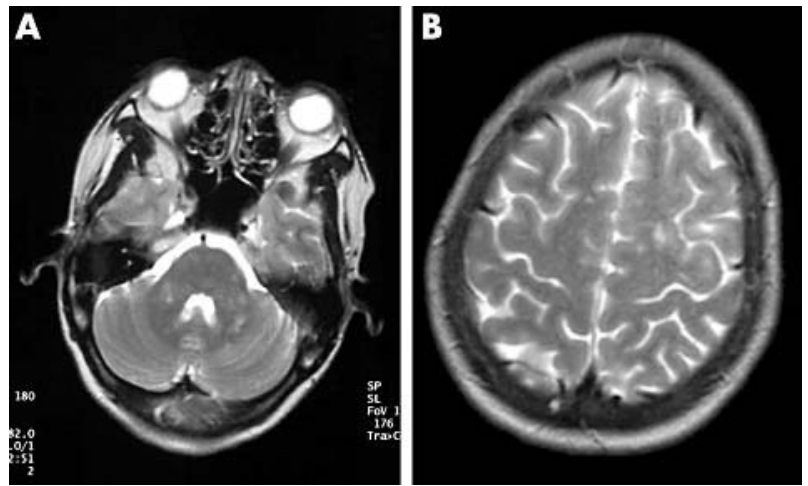

Figure 1 T2 weighted fast spin echo (FSE) images at the level of the pons (A) and over the superior cerebral convexity (B). Focal areas of T2 hyperintensity are present in both middle cerebellar peduncles (A) and in the subcortical white matter of the left superior frontal, middle frontal and precentral gyri. The lesions have no mass effect and are not hypodense on T1 weighted images (not shown). There is no pathological enhancement.

on Tl weighted images progressive multifocal leucoencephalopathy (PML) is less likely.

\section{CASE PRESENTATION}

\section{(Dr Miller and Dr Dave)}

On re-admission, at lumbar puncture the opening pressure was normal. Analysis of CSF showed protein 0.27 and no cells. Syphilis and toxoplasma serology, and CRAG were negative. Polymerase chain reaction (PCR) for human herpes viruses $1-5$ and JC virus was negative. Culture for bacteria, mycobacteria, and fungi was negative.

Could you comment on these developments? What thoughts do you have about the underlying disease process? What investigations are indicated?

\section{DISCUSSION \\ (Dr Wilkins)}

The vomiting and unsteadiness are indicative of cerebellar disease and the right sided pyramidal weakness with intact sensory modalities of a left internal capsular lesion. The right handed dysdiadokokinesis and dysarthria point to right sided cerebellar disease. The unenhanced MRI scan shows multiple small hyperintense lesions in the white matter in and around the cerebellum corresponding to the neurological signs without any surrounding oedema. The lesions are too small for toxoplasmosis or tuberculomata and would be unusual for early PML (also, there was no detectable JC virus DNA in CSF) or multifocal primary CNS lymphoma. If this is an opportunistic infection, the appearances fit best with cryptococcomas but this diagnosis has been excluded. Alternative diagnoses include a vasculitic process (infection or non-infection related), demyelination (again infection or non-infection related), hyperviscosity syndrome, multiple cerebral emboli, or a non-infective granulomatous process (such as sarcoidosis). The retinal appearances are very strange since they appear rapidly but then follow a course that does not parallel her neurological status. Again, it is difficult to ascribe the flame-shaped haemorrhages to an opportunistic infection but they would be in keeping with a diagnosis of cerebral and retinal vasculitis. Hence at this stage, my differential diagnosis includes progressive HIV encephalitis, an immune reconstitution inflammatory syndrome (IRIS) to tuberculosis-given the association with commencing HAART, an as yet unidentified pathogen (although given the array of negative tests this becomes less likely), cerebral vasculitis (possibly secondary to 

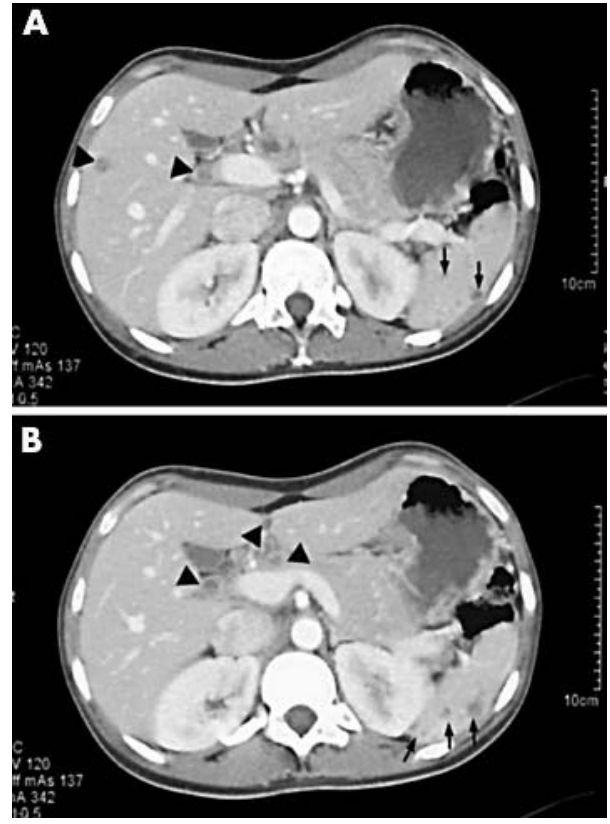

Figure 2 Axial CT through the upper abdomen following oral water and intravenous contrast. (A) There is an ill defined subcentimetre low attenuation lesion in the liver (arrowhead) with multiple smaller lesions scattered throughout the spleen (small arrows). A low attenuation lymph node with peripheral rim enhancement (second arrowhead) is seen in the porta hepatis, lying lateral to the portal vein. (B) Multiple borderline and slightly enlarged, rim enhancing nodes with low attenuation centres are demonstrated in the porta, adjacent to the pancreatic head and anterior to the portal vein (arrowheads). Scattered low attenuation ill defined nodules with smaller satellite lesions are present in a normal sized spleen.

tuberculosis), or demyelination. Investigations that should be considered are MR angiography to demonstrate beading in keeping with a vasculitis, a complete "vasculitis" and hypercoagulable screen, and a full screen for tuberculosis and non-Hodgkin's lymphoma, including a bone marrow aspirate/trephine, and a CT scan of abdomen and thorax).

\section{CASE PRESENTATION \\ (Dr Miller and Dr Dave)}

The retinal appearances were those described either with hypertension or with a hyperviscosity syndrome and the MR images were thought to represent either small vessel disease, possibly vasculitis, or demyelination. Investigations showed the plasma viscosity at 2.2 (normal 1.5-1.7), immunoglobulin electrophoresis showed IgG at $38 \mathrm{~g} / \mathrm{l}$ (normal 7-16), IgA $6.7 \mathrm{~g} / \mathrm{l}$ (normal 1.0-4.0), IgM $3.5 \mathrm{~g} / \mathrm{l}$ (normal 0.4-2.3), and the Coomb's IgG DAT was weakly positive. These results were interpreted as being caused by HIV infection itself. In addition, cryoglobulins were negative, complement $\mathrm{C} 3$ and C4 and lactate dehydrogenase were normal, ANCA antiphospholipid antibodies and hepatitis $\mathrm{C}$ antibody and RNA were negative. An ECG and transthoracic echocardiogram were normal. A bone marrow aspirate and trephine was normocellular, with "reactive" changes. In blood CMV PCR showed a viral load $<100$ copies/ml; HTLV-1 and HTLV-2 antibodies were negative.

Tenofovir was stopped and didanosine was commenced: the patient was now receiving didanosine, lamivudine, and nevirapine. Over the 5 days following readmission the patient's condition deteriorated, with an increase in dysarthria, right sided pyramidal weakness (MRC grade $=3 / 5$ ), and an increased frequency of vomiting. With this background the retinal appearances spontaneously improved. The patient was anticoagulated with heparin, as the presentation was thought possibly to be caused by vasculitis, and vomiting was controlled using a syringe driver to deliver anti-emetics. On day 6 of hospitalisation investigations showed that the arterial blood bicarbonate 17 (normal 22-29) mMol/l, standard bicarbonate was $19 \mathrm{mMol} / \mathrm{l}$ (normal 22-29) and the resting venous lactate $2.8 \mathrm{mMol} / \mathrm{l}$ (normal $<2.2$ ). The patient's antiretroviral therapy was stopped, 9 weeks after it was commenced: nevirapine was discontinued 8 days before didanosine and lamivudine. At this time (early December 2003 ) the CD4 count was 60 cells $\times 10^{6} / 1$.

After 2 weeks anticoagulation was discontinued, as it had no impact on the patient's neurology. A CT scan of the abdomen was performed, as the patient continued to vomit.

\section{IMAGING}

\section{(Dr P J Shaw)}

The abdominal CT was performed after oral water and with intravenous contrast. The main findings are of multiple low attenuation lesions in the spleen, a low attenuation lesion in the liver, and multiple slightly enlarged rim enhancing lymph nodes anterior and lateral to the portal vein (fig 2 ). There are many causes of low attenuation nodules within the liver and spleen, including infections and malignancies. The presence of lymph nodes with peripheral rim enhancement and low attenuation centres following intravenous contrast is useful, as this is a pattern that is more frequently seen with infections, in particular with tuberculosis.

\section{CASE PRESENTATION}

\section{(Dr Miller and Dr Dave)}

Would you comment on the CT findings? What further investigations are indicated?

\section{DISCUSSION \\ (Dr Wilkins)}

The abdominal CT scan demonstrates several low attenuation lesions in the liver and spleen. The para-aortic lymph nodes are enlarged and show central necrosis. Given her worsening clinical condition, it is imperative to obtain tissue for histological analysis to try and pin down what is going on. The assumption has to be that whatever is going on in the abdomen is the same or closely linked with the pathological process in her brain. An ultrasound guided liver or lymph node biopsy would be indicated. The negative vasculitis screen does not support a non-infective cause of vasculitis, although the possibility that this is tuberculosis, especially with the demonstration of the CT scan abnormalities, pushes me towards this being the diagnosis with complicating cerebral vasculitis and probably a component of IRIS, given the temporal association with commencing HAART.

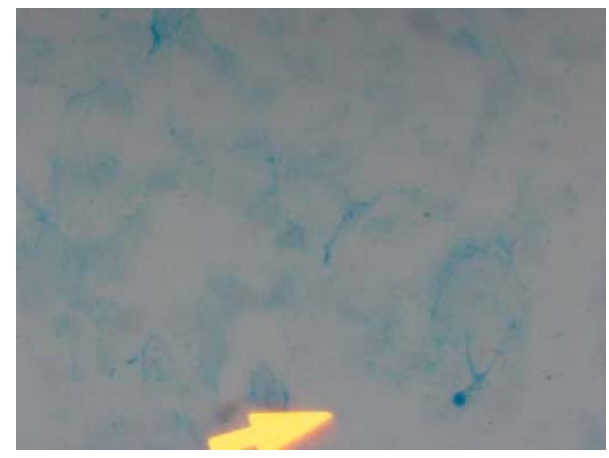

Figure 3 Fine needle aspirate smear showing scanty AAFB (arrow): there are no epitheloid cells. Zeihl-Neelsen stain. Magnification $\times 100$. 


\section{CASE PRESENTATION}

\section{(Dr Miller and Dr Dave)}

A percutaneous fine needle aspirate of an abdominal lymph node was performed. The aspirate showed coagulative necrosis, but no granulomata formation, or epitheloid cells: scanty alcohol and acid fast bacilli (AAFB) were seen (fig 3). SDA and subsequent culture identified the organism as fully sensitive Mycobacterium tuberculosis. At this time culture of two CSF samples, blood, bone marrow, urine, and stool was negative for mycobacteria.

How would you treat the tuberculosis?

\section{DISCUSSION \\ (Dr Wilkins)}

Given that this is $M$ tuberculosis and there is no evidence of drug resistance, the optimum treatment would be rifampicin based and include pyrazinamide, ethambutol, and isoniazid, with a plan to treat for at least 9 months as the patient possibly has cerebral disease. Once antiretroviral treatment commences, there may need to be a switch in her antituberculosis treatment because of potential drug-drug interactions. It is worth emphasising the importance of using molecular techniques to confirm that this is tuberculosis and that the strain is rifampicin sensitive (a negative rifampicin probe is $90 \%$ predictive of the absence of rifampicin resistance). A surprising number of cultures had been taken from this patient and yielded no growth suggesting that an unusual atypical mycobacterium such as $M$ genevensae may have been the cause if the AAFB had not been confirmed to be $M$ tuberculosis by probe and culture.

\section{CASE PRESENTATION}

\section{(Dr Miller and Dr Dave)}

The patient was treated with rifampicin, isoniazid, pyrazinamide, and ethambutol, together with vitamin $\mathrm{B}_{6}$. There was concern about drug absorption, as the patient continued to vomit. A repeat MR scan of the brain was performed in midDecember 2003, 4 weeks after the original MRI.

\section{IMAGING}

\section{(Dr Jäger)}

There has been progression of the infratentorial white matter lesions, which parallel the clinical progression. The differential diagnosis remains unchanged (fig 4).
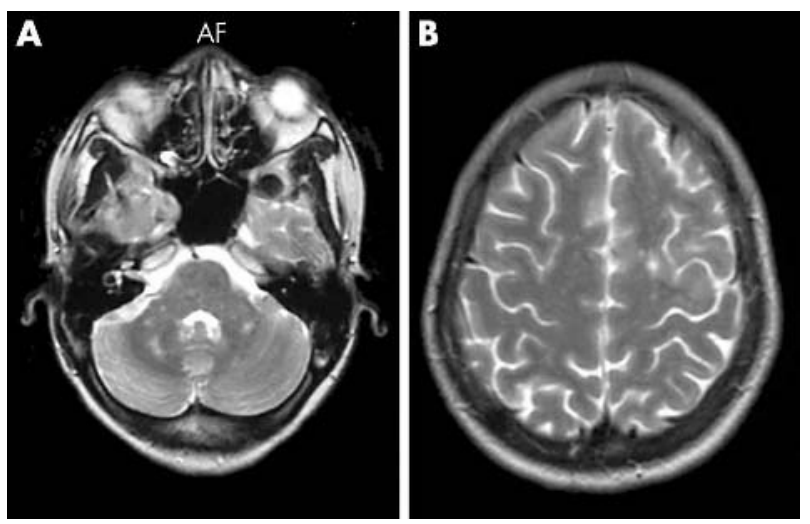

Figure 4 T2 weighted fast spin echo (FSE) images at the level of the pons (A) and over the superior cerebral convexity (B). Compared to the images shown in figure 1 there has been progression of the infratentorial white matter lesions (A) but the infratentorial lesions have not changed significantly (B).

\section{CASE PRESENTATION}

\section{(Dr Miller and Dave)}

A repeat lumbar puncture showed in CSF a normal opening pressure, no cells, protein at $0.3 \mathrm{~g} / \mathrm{l}$, and PCR was negative for human herpes viruses $1-5$ and 6-7, and JC virus. In blood serology for arboviruses (alphaviruses, flaviviruses, and phleboviruses) and Lyme was negative. The patient continued to deteriorate neurologically.

As the follow up MRI suggested demyelination the patient was treated empirically with intravenous methylprednisolone ( $0.5 \mathrm{~g} /$ day, for 5 days). This intervention had no impact on the patient's symptomatology and she continued to deteriorate over the next 10 days. Re-examination at this stage showed new signs. The patient had developed a right upper motor neuron fifth cranial nerve lesion, bilateral horizontal gaze palsy, and she was unable to verbalise. There was no gag or swallow reflex. In the limbs there was a right sided pyramidal weakness $(\mathrm{MRC}=$ grade $2 / 5$ in the arm and $4 / 5$ in the leg). She had generalised hypertonia/hyper-reflexia. The patient received feeding and drugs through a nasogastric tube. Over the next 14 days the patient displayed signs of recovery. There was an obvious improvement in her speech and the pyramidal weakness in the arm partially resolved $(\mathrm{MRC}=4 / 5)$.

In early January 2004 HAART was restarted (using didanosine, lamivudine, and nevirapine) and the antituberculosis therapy was modified (rifampicin was changed to rifabutin), in order to reduce the likelihood of drug-drug interactions. Within 4 days the patient became less well. She described an increase in headache, was drowsier and was agitated and tearful. She denied nightmares or confusion. Over the next 10 days there was a continued deterioration in the patient's symptoms. There was no deterioration in the signs in the cranial nerves or limbs. A repeat cranial MRI scan was performed.

\section{IMAGING}

\section{(Dr Jäger)}

There has been a dramatic deterioration in the MR appearances, with an increase in size of the infratentorial
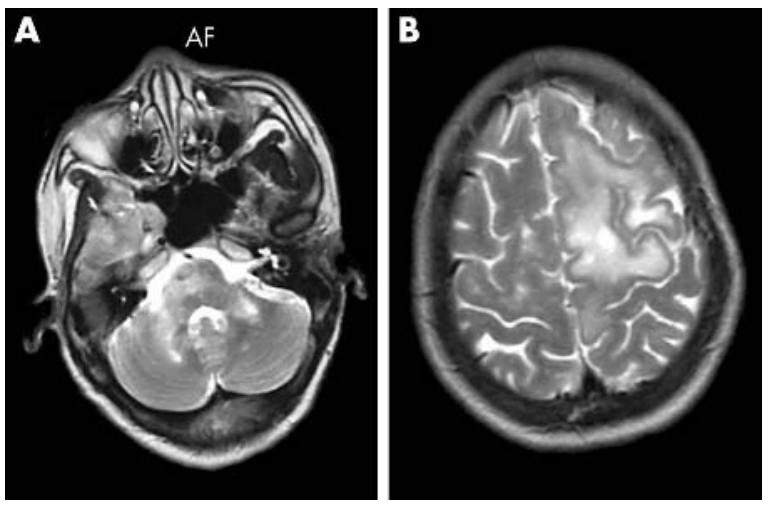

Figure 5 T2 weighted fast spin echo (FSE) images at the level of the pons (A) and over the superior cerebral convexity (B). The T2 hyperintense lesions are much more extensive, when compared to the appearances shown in figure 4 and additionally have become more confluent. The infratentorial lesions $(A)$ involve a large part of both middle cerebral peduncles and extend into the pons, more severely affecting the right side. Further images (not shown) demonstrate involvement of the medulla oblongata and more widespread involvement of the cerebellar white matter. Supratentorially (B) the confluent T2 hyperintensities involve most of the white matter of the left superior, middle, and precentral gyri, sparing a thin mantle of grey matter. Despite marked progression there is only little local mass effect with slight expansion of the gyri. There is no hypointensity on $\mathrm{Tl}$ weighted images, or pathological enhancement. 
and supratentorial white matter lesions (fig 5). These lesions still do not show significant hypointensity on Tl weighted imaging and PML remains an unlikely diagnosis. A demyelinating process producing lesions of this size would have to be highly active and would normally be associated with pathological enhancement, particularly in the case of acute disseminated encephalomyelitis (ADEM). The rapid progression is compatible with a vasculitic process. The sensitivity of angiography for diagnosis of cerebral vasculitis depends on the size of the arteries involved. A proximal vasculitis affecting the major branches of the intracranial vessels may be diagnosed frequently by use of non-invasive techniques, including MR angiography. By contrast, a vasculitic process affecting small vessels of the brain, including those supplying the white matter, may be very difficult to diagnose even with conventional digital subtraction angiography (DSA), as the size of the vessels is beyond the resolution of DSA. Cerebral vasculitis is best confirmed by cerebral biopsy.

\section{CASE PRESENTATION (Dr Miller and Dr Dave)}

These findings raised the possibility that the patient might have PML. A lumbar puncture was performed. CSF analysis showed normal protein and glucose, and no cells. PCR analysis showed JC virus DNA was not detected, but EBV DNA was detected.

\section{VIROLOGY}

\section{(Dr J W Tang)}

In blood EBV VCA was $\operatorname{IgG}^{+}$and $\operatorname{IgM}^{-}$, and anti-EBNA was positive on samples from November 2003, December 2003, and January 2004. These results suggest a past primary EBV infection and the detectable EBV DNA in blood is most likely the result of detection of latent EBV infection of lymphocytes, or to reactivation of EBV. Results of quantitative PCR for EBV DNA performed on peripheral blood are shown in table 1 .

\section{CASE DISCUSSION \\ (Dr Miller and Dr Dave)}

How do you interpret these findings? What would you do now?

\section{DISCUSSION \\ (Dr Wilkins)}

The changes on cranial MRI are in the white matter and would be consistent with demyelination, as in PML. The deterioration in both clinical and imaging appearances after commencing HAART is consistent with reports of IRIS in this condition. ${ }^{1}$ Nevertheless, on three occasions PCR on CSF for $\mathrm{JC}$ virus has been negative and the progression in the scans and clinical status would be unusual for PML. The rise in plasma titre of EBV DNA is consistent with non-specific reactivation, possibly related to $\mathrm{HAART}^{2}$ and given the MR appearances and progression, as well as the previously negative qualitative tests, I do not believe CNS lymphoma

Table 1 Plasma EBV DNA, measured by quantitative PCR

\begin{tabular}{lcl}
\hline Date & Copies/ml & $\begin{array}{l}\text { Patient taking } \\
\text { HAART }\end{array}$ \\
\hline 10 Sept 03 & 5 & No \\
01 Oct 03 & 2983 & Yes \\
23 Oct 03 & 3932 & Yes \\
23 Dec 03 & 761 & No \\
15 Apr 04 & 71 & No \\
\hline
\end{tabular}
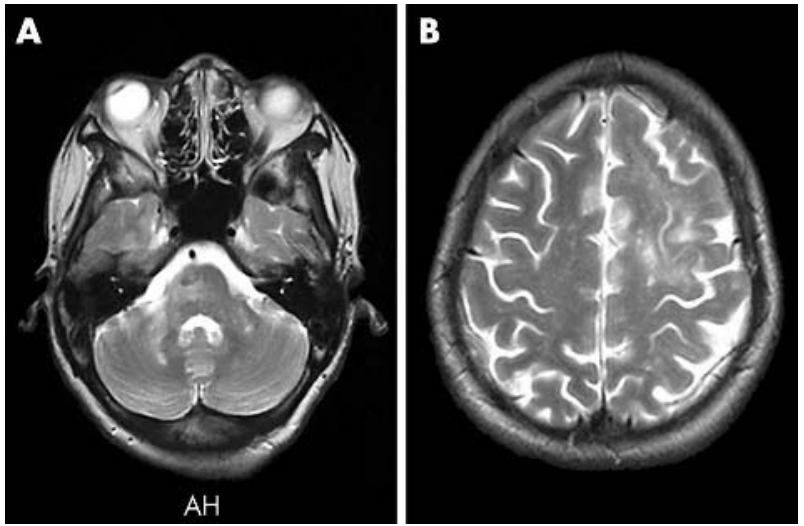

Figure 6 T2 weighted fast spin echo (FSE) images at the level of the pons (A) and over the superior cerebral convexity (B). Compared to the appearances shown in figure 5 the appearances on T2 weighed imaging are much less extensive and have more clearly defined boundaries. The slight swelling of the left superior convexity gyrus has also resolved. The majority of the lesions were now hypodense on $\mathrm{Tl}$ weighted imaging (images not shown)

is a sustainable diagnosis. I would petition the neurosurgeons to perform a brain biopsy and would also continue the treatment for tuberculosis, as there is positive proof for this diagnosis. I would not give further steroids because of my slight concern that it may mask a co-existent lymphoma or pre-malignant vasculitic condition such as lymphomatoid granulomatosis. ${ }^{3}$ I would also discontinue HAART because of the rapid deterioration in her clinical status, which I would do abruptly and not staggered, despite the risk of promoting non-nucleoside reverse transcriptase inhibitor (NNRTI) resistance. In my opinion, her neurological deterioration overrides this concern.

\section{CASE DISCUSSION}

\section{(Dr Miller and Dr Dave)}

The patient received empirical cidofovir, given the progressive neurological deterioration and the concerns about possible PML. HAART was stopped, after 2 weeks of therapy, and antituberculosis therapy was stopped, after 2.5 months of therapy.

Over the next 3-4 weeks there was objective improvement in the patient's speech (she was markedly less dysarthric) and in the power in her right arm (she was able to transfer from bed to chair with help from one person). A repeat MR image was performed in March 2004 (interval since the last scan 6 weeks).

\section{IMAGING \\ (Dr Jäger)}

The appearances on MRI are much improved. The T2 weighted hyperintense lesions are much less extensive (fig 6). These MR appearances are compatible with the chronic phase of an inflammatory or vasculitic process.

\section{CASE PRESENTATION}

\section{(Dr Miller and Dr Dave)}

A further lumbar puncture was performed. PCR on CSF again showed EBV DNA: PCR for enterovirus RNA was negative. In blood a vasculitis screen (as above) was negative. A CD4 count was 80 cells $\times 10^{6} / \mathrm{l}$. At the end of March 2004 the patient was transferred to a neurorehabilitation unit. At this stage she was able to walk with the aid of one person, she had obvious dysarthria and displayed no emotional lability. Antituberculosis therapy was restarted. 
Could you suggest a working diagnosis? For how long would you treat the tuberculosis? When and with what drugs would you start HAART?

\section{DISCUSSION (Dr Wilkins)}

The diagnosis of abdominal tuberculosis involving the paraaortic lymph nodes is incontrovertible. Given the presenting pretreatment CD4 count of $<50$ cells $\times 10^{6} / 1$, the disease is likely to be disseminated and involve the CNS despite the absence of pleocytosis or biochemical derangement of the CSF. The first MR scan is consistent with a vasculitic process, which is a well recognised, albeit rare, complication of tuberculosis. The subsequent deterioration in her clinical status and the development of significant white matter change on the next two MR scans is consistent with an immune mediated process to the underlying pathogen, and probable demyelination. Given the confirmation of abdominal tuberculosis, I believe the likely cause of her illness to be disseminated tuberculosis with CNS involvement complicated by cerebral vasculitis and an IRIS syndrome. Although there is a significant increase in the plasma EBV DNA levels simultaneous to her HAART treatment, I think this is more likely to represent a non-significant reactivation ${ }^{2}$ as opposed to CNS lymphoma, lymphomatoid granulomatosis, or EBV encephalitis or EBV related IRIS.

I would treat the tuberculosis for a minimum of 9 months assuming that a rifamycin was part of the regimen. If not, treatment should be continued for 18-24 months and only stopped when there is clinical, radiological, and inflammatory marker evidence of inactivity. ${ }^{4}$ With respect to the timing of HIV treatment, this is always a contentious issue. On the one hand, much of this woman's morbidity is a consequence of starting antiretroviral therapy and on two occasions it needed to be discontinued. However, she also runs a risk of significant other illness relating to her HIV if antiretroviral therapy is delayed too long and there is evidence that the mortality rate is higher in those where HIV treatment is delayed. ${ }^{4}$ Before commencing HIV treatment, an HIV resistance test should be performed, especially as the previous regimens contained efavirenz and lamivudine; both drugs have a low genetic barrier to resistance. In the context of a rifamycin-containing regimen, the recommendation, assuming wild type HIV, is efavirenz with two nucleoside reverse transcriptase inhibitors (NRTI), using a higher dose of efavirenz. However, this patient's previous experience and current neurological status indicates that this would be an unwise choice, rendering it difficult to distinguish drug toxicity from neurological deterioration. One option is to recommence the nevirapine (with therapeutic drug monitoring) although there are scant data for doing this. Triple NRTI regimens have been proved to be suboptimal in potency and therefore the option I would go for is to use a protease inhibitor and switch from rifampicin to rifabutin. My choice would be a combination of lopinavir/ritonavir, tenofovir, and emtricitabine with a switch to rifabutin, given three times a week, as the protease inhibitor increases levels of rifabutin. Given the patient's improving and stable clinical status I would recommence therapy straight away.

\section{CASE PRESENTATION}

\section{(Dr Miller and Dr Dave)}

By June 2004 the patient was walking with the aid of a stick and her dysarthria was much improved. In the right arm there was a residual $4 / 5$ pyramidal weakness. At this time the CD4 count 20 cells $\times 10^{6} / 1$ and HIV viral load 40330 copies/ $\mathrm{ml}$. In mid-July the patient began HAART, with lamivudine, tenofovir, and lopinavir: the dose of rifabutin was reduced to compensate for the introduction of lopinavir. In August 2004 the patient was walking unaided; the dysarthria and arm weakness were unchanged. The antituberculosis therapy was stopped in September 2004, the patient having completed 6 months of treatment. At this time the CD4 count 80 cells $\times 10^{6} / \mathrm{l}$ and HIV viral load 50 copies $/ \mathrm{ml}$.

\section{DISCUSSION}

\section{(Dr Wilkins)}

The differential diagnosis of neurological disease in an HIV infected patient with advanced immune compromise is wide and includes classic opportunistic infections and HIV related tumours. However, immune reconstitution with HAART can result in paradoxical changes in focal lesions and also demyelinating syndromes.

Through restoration of pathogen specific immune responses, HAART may be accompanied by atypical presentations of the classic opportunistic pathogens. When occurring early on after initiating HAART it usually reflects an immunorestorative response (usually termed IRIS) to a recently diagnosed infection for which the patient has commenced treatment but has not completed the course, or a quiescent but low grade active but undiagnosed infection. When it occurs later, the response is directed to antigen from non-viable organisms. It therefore represents an unwelcome marker of a successful response to therapy. However, if not recognised as such and managed appropriately, the consequences may be severe with significant morbidity and occasional mortality. Risk factors for IRIS include a low CD4 nadir $\left(<50\right.$ cells $\left.\times 10^{6} / 1\right)$, a rapid rise in CD4 count and drop in viral load, and early introduction of HAART after treatment of an AIDS defining opportunistic infection. The most frequently implicated pathogens are Mycobacterium avium complex (MAC), ${ }^{56} \mathrm{M}$ tuberculosis, ${ }^{56} \mathrm{CMV}, \mathrm{VZV}$, and Cryptococcus neoformans. Less frequent but well characterised opportunists include JC virus causing PML, ${ }^{1}$ Pneumocystis jirovecii, and hepatitis $\mathrm{B}^{7}$ and $\mathrm{C}$ viruses. MAC characteristically presents with fevers, inflammatory lymphadenitis/focal collections, or pulmonary infiltrates during the first 3 months of treatment. Blood cultures are usually negative but the organisms can sometimes be isolated from the focal lesion. $M$ tuberculosis related IRIS comes on earlier (from 1-4 weeks) as a rule, with a variety of presentations partly dependent on the site(s) of the original disease. Fever is nearly always present and lymphadenitis often (peripheral or central). Low grade CMV infection may first announce itself with IRIS related retinitis or uveitis/vitritis, or it may represent a relapse of established and treated disease (with or without maintenance anti-CMV therapy). It is more common in patients with more extensive CMV retinitis and can result in significant and chronic eye damage requiring suppressive intraorbital steroids.

Management of IRIS is always tricky but in general entails continuation of HAART, continuation of the therapy/prophylaxis for the opportunistic infection, and use of non-steroidal anti-inflammatory drugs or corticosteroids to suppress the immune mediated pathogen specific response. Rarely, as in this case, it is essential to discontinue the antivirals because of the failure to respond to corticosteroids and the anatomical site of the IRIS. Management of IRIS in MAC or M tuberculosis is particularly difficult because of the need for a prolonged treatment course of antituberculosis drugs including a rifamycin where possible, the need at some time to introduce HAART, and the potential drug-drug interactions with rifamycins, the difficulty of making the diagnosis accurately and knowing whether the finding of AAFB indicates live or dead bacilli, and the severity of the clinical disease that can result from mycobacterial associated IRIS.

Detection of viral DNA in CSF by PCR has had a major impact in the diagnosis of CNS infection in both immune 
competent and compromised patients. ${ }^{8}$ With HIV infected patients living longer and the classic opportunistic infections decreasing in incidence or presenting in less typical ways, neurological complications have become a major and challenging problem in HIV. In many circumstances, they have become the non-invasive investigation of choice supplanting the requirement for brain biopsy. The advantages of PCR for DNA detection in CSF is that the test is rapid, more sensitive than standard techniques for diagnosis, allows identification of micro-organisms that cannot be cultured or are fastidious in their growth requirements and, in certain instances, can be quantified to determine progression of a condition (such as with JC virus). As with viral culture or serology, results depend on when the CSF sample was taken in relation to the onset of disease, and the initiation of any specific therapy. Lastly, demonstration of specific DNA by PCR allows identification of unusual presentations of neurological infections. Disadvantages are a lower specificity (although most PCR tests have specificities over 95\%), and the potential for sample contamination. For HSV-1 and HSV2, the demonstration of DNA in the CSF has replaced the need for biopsy in patients with suspected herpes encephalitis, having a sensitivity of $>90 \%$, and a specificity approaching 100\%. For detection of JC virus DNA, sensitivity is lower (approximately 75\%; specificity $92-100 \%$ ) but again its detection obviates the need for biopsy in most cases where MR findings are characteristic. With treatment of PML, JC virus DNA levels fall rapidly in CSF and the test can be used to monitor the disease. In the majority of HIV infected patients in whom cranial MR imaging demonstrates a single or $<5$ mass lesions, detection of EBV DNA in CSF is indicative of primary CNS lymphoma (>90\% sensitivity and specificity). ${ }^{9}$ In this particular patient, the negative results on CSF on repeated occasions were particularly helpful.

Both vasculitis and demyelination can give rise to the diffuse white matter changes seen on the MR scans. Vasculitis is a broad term representing an inflammatory process of blood vessels and many of these conditions can affect the cerebral vessels. Classification of the major groups is into systemic vasculitis (such as polyarteritis nodosa and those secondary to infection), hypersensitivity vasculitis (for example, Henoch Schonlein purpura), systemic granulomatous disorders (for example, lymphomatoid granulomatosis), giant cell arteritis, connective tissue disorders (for example, systemic lupus), and paraneoplastic. Those infections associated with HIV, which can be complicated by CNS vasculitis, include tuberculosis, syphilis, varicella zoster, and cryoglobulinaemia associated hepatitis C. Rarely, HIV itself has been identified as a cause of necrotising vasculitis and granulomatous angiitis: CNS lymphoma is another uncommon association.

Another possibility that needs to be entertained is a demyelinating process. On MR imaging, the appearances of ADEM are very similar to PML. The absence of JC virus in CSF and the evolution and subsequent improvement without effective treatment for the condition attest against PML and raise the possibility of another organism causing the demyelinating process. In cases of infection related ADEM, the absence of viral particles in the brain, the occurrence in the context of a resolving illness, and the improvement with high dose steroids favour an immunologically mediated process. This is consistent with the temporal association with HAART in this patient. Although HIV has been identified as a cause of ADEM, the few cases that have been described have been at higher CD4 counts, suggesting that this patient's illness was secondary to another pathogen. EBV is a recognised CNS pathogen in immune competent as well as compromised people. ${ }^{10}$ The identification of moderately high titres of plasma EBV DNA at the time white matter changes were demonstrated on cranial MR imaging raises the possibility that EBV was the cause of a post-infectious demyelinating process resulting from immune reconstitution.

Without brain histology, a definitive diagnosis cannot be made. Certainly, this patient had culture positive fully sensitive $M$ tuberculosis. Her neurological progression with corresponding MR changes and association with HAART and a good immunological response argues strongly for an immune mediated aetiology. The only other positive information on investigation is a temporal association between her white matter changes and rising and subsequent falling plasma EBV DNA titres.

I think she has had tuberculosis with probable CNS involvement and possible vasculitis. She has also had complicating CNS IRIS secondary either to EBV or tuberculosis or possibly both. The severity and complexity of her illness underscores the necessity for brain histology in cases such as this.

\section{ACKNOWLEDGEMENTS}

We thank Mary Fazalon, Department of Histopathology, University College London Hospitals for reporting FNA cytology.

\section{Authors' affiliations}

R F Miller, S S Dave, Patrick Manson Unit, University College London Hospitals, and Mortimer Market Centre, Camden PCT, London WCIE 6AU, UK

JW Tang, Department of Virology, University College London Hospitals, London WIT 4JF, UK

H R Jäger, P J Shaw, Department of Imaging, University College London Hospitals, London WC1E 6DB, UK

E G L Wilkins, Department of Infectious Diseases, North Manchester General Hospital, Manchester M8 5RB, UK

\section{REFERENCES}

1 Safdar A, Rubocki RJ, Horvath JA, et al. Fatal immune restoration disease in human immunodeficiency virus type-1-infected patients with progressive multifocal leukoencephalopathy: impact of antiretroviral therapy-associated immune reconstitution. Clin Infect Dis 2002;35:1250-7.

2 Righetti E, Ballon G, Ometto L, et al. Dynamics of Epstein-Barr virus in HIV-1infected subjects on highly active antiretroviral therapy. AIDS 2002;16:63-7.

3 Mizuno T, Takanashi Y, Onodera H, et al. A case of lymphoid granulomatosis/angiocentric immunoproliferative lesion with long clinical course and diffuse brain involvement. J Neurol Sci 2003;213:67-76.

4 Pozniak AL, Miller RF, Lipman MCl, et al. BHIVA guidelines for the treatment of TB/HIV co-infection. HIV Medicine 2005; (in press).

5 Buckingham SJ, Haddow $\amalg$, Shaw PJ, et al. Immune reconstitution inflammatory syndrome in HIV-infected patients with mycobacterial infections starting highly active anti-retroviral therapy. Clin Radiol 2004;59:505-13.

6 Wendel KA, Alwood KS, Gachuhi, et al. Paradoxical worsening of tuberculosis in HIV-infected persons. Chest 2001;120:193-7.

7 Carr A, Cooper DA. Restoration of immunity to chronic hepatitis infection in HIV-infected patients on protease inhibitor. Lancet 1997;349:995-6.

8 Fox JD, Brink NS, Zuckerman MA, et al. Detection of herpesvirus DNA by nested PCR in the CSF of HIV infected individuals with neurological disease: a prospective evaluation. J Infect Dis 1995;172:1087-90.

9 Brink NS, Sharvell Y, Howard MR, et al. Detection of Epstein-Barr virus and Kaposi's sarcoma-associated herpesvirus DNA in CSF from persons infected with HIV who had neurological disease. J Neurol Neurosurg Psychiat 1998;65:191-5.

10 Fujimoto $\mathbf{H}$, Asaoka K, Imaizumi T, et al. Epstein-Barr virus infections of the central nervous system. Intern Med 2003;42:33-40. 